\title{
Assessment of Myocardial Viability in Patients with Heart Failure*
}

\author{
Arend F.L. Schinkel ${ }^{1}$, Don Poldermans ${ }^{1}$, Abdou Elhendy ${ }^{2}$, and Jeroen J. Bax ${ }^{3}$ \\ ${ }^{I}$ Thoraxcenter, Department of Cardiology, Erasmus Medical Center, Rotterdam, The Netherlands; ${ }^{2}$ Department of Cardiology, \\ Marshfield Clinic, Marshfield, Wisconsin; and ${ }^{3}$ Department of Cardiology, Leiden University Medical Center, Leiden, The Netherlands
}

The prognosis for patients with chronic ischemic left ventricular dysfunction is poor, despite advances in different therapies. Noninvasive assessment of myocardial viability may guide patient management. Multiple imaging techniques have been developed to assess viable and nonviable myocardium by evaluating perfusion, cell membrane integrity, mitochondria, glucose metabolism, scar tissue, and contractile reserve. PET, ${ }^{201} \mathrm{TI}$ and $99 \mathrm{~m}$ Tc scintigraphy, and dobutamine stress echocardiography have been extensively evaluated for assessment of viability and prediction of clinical outcome after coronary revascularization. In general, nuclear imaging techniques have a high sensitivity for the detection of viability, whereas techniques evaluating contractile reserve have a somewhat lower sensitivity and a higher specificity. MRI has a high diagnostic accuracy for assessment of the transmural extent of myocardial scar tissue. Patients with a substantial amount of dysfunctional but viable myocardium are likely to benefit from coronary revascularization and may show improvements in regional and global contractile function, symptoms, exercise capacity, and long-term prognosis.

Key Words: myocardial viability; heart failure; noninvasive imaging; prognosis; stunning; hibernation

J Nucl Med 2007; 48:1135-1146

DOI: 10.2967/jnumed.106.038851

C hronic heart failure is becoming the main clinical challenge in cardiology in terms of the number of patients involved. Over the last decade, the number of patients with this clinical syndrome has increased considerably; recent estimations have shown that 5 million patients in the United States have chronic heart failure, with 550,000 new patients being diagnosed annually, resulting in over 1 million hospitalizations (1). The diagnostic and therapeutic costs involved are estimated to be more than $\$ 29$ billion per year (1). The major portion of these costs appears to be related

Received Dec. 11, 2006; revision accepted Apr. 9, 2007.

For correspondence or reprints contact: Jeroen J. Bax, Department of Cardiology, Leiden University Medical Center, Albinusdreef 2, 2333 ZA Leiden, The Netherlands.

E-mail: j.j.bax@lumc.nl

*NOTE: FOR CE CREDIT, YOU CAN ACCESS THIS ACTIVITY THROUGH THE SNM WEB SITE (http://www.snm.org/ce_online) THROUGH JULY 2008.

No potential conflict of interest relevant to this article was reported.

COPYRIGHT @ 2007 by the Society of Nuclear Medicine, Inc. to the (recurrent) hospitalizations of these patients for decompensating heart failure.

The most important cause of heart failure is chronic coronary artery disease. Gheorghiade and Bonow (2) pooled data from 13 randomized, multicenter heart failure drug trials (involving over 20,000 patients) reported in The New England Journal of Medicine between 1986 and 1997. The authors concluded that coronary artery disease was the underlying etiology in almost $70 \%$ of the patients. The actual figure may have been even higher because many patients in these trials did not undergo coronary angiography.

The long-term prognosis for patients with heart failure remains poor, despite advances in different therapies. Recent data from the Framingham Heart Study demonstrated 5 -y mortality rates of $59 \%$ for men and $45 \%$ for women with heart failure in the period from 1990 through 1999 (3). Mortality rates are particularly high in older people with heart failure.

\section{THERAPEUTIC OPTIONS FOR ISCHEMIC HEART FAILURE}

Currently, several therapeutic options are available for patients with ischemic cardiomyopathy (Table 1). Progress in pharmacologic therapy has contributed considerably to the survival of patients with heart failure. Device therapy with biventricular pacing and internal cardiac defibrillators may further improve outcome in selected patients. Heart transplantation is associated with a good long-term prognosis, but the number of donor hearts is limited, and many patients die awaiting transplantation. Some patients benefit from a left ventricular (LV) assist device as a temporary treatment before transplantation. Revascularization is an alternative therapy, although the associated risk is increased in patients with a severely depressed LV ejection fraction (LVEF). Moreover, not all patients with ischemic cardiomyopathy show improvement in contractile function after revascularization; approximately one third of dysfunctional segments improve in function, and approximately $40 \%$ of patients show improvement in the LVEF (4).

Therefore, in view of the high morbidity and mortality associated with revascularization procedures, careful selection 
TABLE 1

Main Therapeutic Options for Ischemic Cardiomyopathy

\begin{tabular}{cl}
\hline \multicolumn{1}{c}{ Option } & \multicolumn{1}{c}{ Example or characteristic } \\
\hline Medical therapy* & Diuretics \\
& Digoxin \\
& Angiotensin-converting enzyme \\
inhibitors & Angiotensin II receptor blockers \\
& $\beta$-Blockers \\
& Spironolactone \\
& Amiodarone \\
& Biventricular pacemaker \\
& Internal cardiac defibrillator \\
& LV assist device (as bridge to \\
Device therapy & transplantation) \\
& Limited number of donor hearts does \\
& not meet large demand \\
Heart & Excessive comorbidity in potential \\
transplantation & recipients \\
& Good long-term survival \\
& Revascularization if viable myocardium \\
is present, but associated risk is high & Mitral valve repair \\
Surgery & LV aneurysmectomy \\
& LV restoration
\end{tabular}

${ }^{*}$ Despite new drugs, survival of patients receiving medical therapy is poor.

of patients who may benefit from revascularization procedures appears to be warranted. Over the last 2 decades, evidence has been collected that patients with dysfunctional but viable myocardium are likely to benefit from revascularization, whereas patients without viable myocardium will not benefit.

Various investigators using different imaging techniques have reported on the incidence of viability in patients with chronic ischemic LV dysfunction (Table 2) (5-10). In general, viability was detected in more than $50 \%$ of the dysfunctional segments. This observation emphasizes that it is clinically relevant to search for viability in patients with chronic ischemic cardiomyopathy.

TABLE 2

Viability in Patients with Chronic Ischemic LV Dysfunction

\begin{tabular}{ccclc}
\hline Reference & $\begin{array}{c}\text { No. of } \\
\text { patients }\end{array}$ & $\begin{array}{c}\text { Mean } \pm \text { SD \% } \\
\text { LVEF }\end{array}$ & $\begin{array}{c}\text { Imaging } \\
\text { technique }\end{array}$ & $\%$ Viability \\
\hline 5 & 27 & $19 \pm 6$ & PET & 52 \\
6 & 283 & $26 \pm 8$ & PET & 55 \\
7 & 104 & $25 \pm 7$ & SPECT & 61 \\
8 & 27 & NA & SPECT & 37 \\
9 & 150 & $31 \pm 12$ & DSE & 58 \\
10 & 387 & $30 \pm 11$ & SPECT & 58
\end{tabular}

DSE = dobutamine stress echocardiography; NA = not available.

\section{Definition of Myocardial Viability}

The concept of myocardial hibernation was introduced by Rahimtoola to describe a condition of chronic sustained abnormal contraction attributable to chronic underperfusion in patients who have coronary artery disease and in whom revascularization causes the recovery of LV function (11). Myocardial stunning has been defined as reversible myocardial contractile dysfunction in the presence of normal resting myocardial blood flow $(12,13)$. The brief regional myocardial functional and electrophysiological alterations related to myocardial stunning were initially observed after brief coronary artery occlusion in dogs (12). Myocardial hibernation and myocardial stunning are pathophysiologic entities that may coexist in patients with ischemic cardiomyopathy. Vanoverschelde et al. (14) suggested that repeated ischemic attacks may induce chronic dysfunction in the presence of normal or mildly reduced resting perfusion; this condition was referred to as repetitive stunning. It appears that there is a temporal progression from stunning, characterized by (nearly) normal flow (with reduced flow reserve), to hibernation, with reduced resting flow.

Myocardial hibernation and stunning are not merely pathophysiologic concepts. Elsasser et al. (15) demonstrated signs of energy depletion and downregulation of energy turnover in hibernating myocardium. The alterations in energy metabolism may trigger and maintain contractile dysfunction, continuous tissue degeneration, and cardiomyocyte loss. Studies in patients with ischemic cardiomyopathy showed structural dedifferentiation of cardiac myocytes in biopsy samples obtained at the time of coronary revascularization (16). Hibernating myocardium showed a loss of contractile filaments (sarcomeres), an accumulation of glycogen in the spaces previously occupied by the myofilaments, nuclei with uniformly distributed chromatin, small mitochondria, and a nearly absent sarcoplasmic reticulum (16).

Several noninvasive techniques have been developed to detect signs of viability, such as an intact cell membrane, residual glucose metabolism, or preserved contractility in response to dobutamine stimulation. Because hibernating myocardium represents a delicate balance among flow, function, and viability and because myocytes adapt their activity level to prevailing circumstances, it is likely that some characteristics (e.g., contractile reserve) are lost while more basal characteristics, such as glucose metabolism and cell membrane integrity, are preserved. Additionally, in some patients, the increased demands of inotropic stimulation will exceed the limited flow reserve and result in myocardial ischemia (17). This situation may explain, in part, the reduced sensitivity of imaging techniques focusing on contractile reserve to detect viable but hypoperfused myocardium in comparison with the sensitivity of perfusion imaging (17).

\section{Endpoints in Viability Studies}

Currently available studies evaluating the role of noninvasive imaging techniques in the assessment of myocardial 
viability have focused on various clinical endpoints. The endpoints used in viability studies after revascularization include improvement in regional LV function (segments), improvement in global LV function (LVEF), improvement in symptoms (New York Heart Association [NYHA] functional class), improvement in exercise capacity (metabolic equivalents), reverse LV remodeling (LV volumes), prevention of sudden death (ventricular arrhythmias), and longterm prognosis (survival). Improvement in function after revascularization is still considered the final proof of viability. In a recent analysis of pooled data, including 105 studies (with 3,003 patients) that focused on viability assessment (with nuclear imaging and dobutamine stress echocardiography), 15,045 dysfunctional segments were analyzed for viability with noninvasive testing; 7,941 segments $(53 \%)$ showed improvement in function after revascularization (18). Of these 7,941 segments with improvement in function, $84 \%$ were considered to be viable according to the imaging modalities. From a clinical point of view, improvement in global LV function (LVEF) may be more important than improvement in regional function. The LVEF has been demonstrated to be a very powerful predictor of prognosis. However, the majority of imaging studies focusing on viability assessment have evaluated only segmental improvement. A few studies have examined viability in relation to a change in the LVEF; these studies consistently showed that patients with a substantial amount of viable tissue showed improvement in the LVEF after revascularization. The precise proportions of viable segments needed to result in improvement in the LVEF differed among the studies, and it is currently unclear how much viability is needed to result in improvement in the LVEF after revascularization. The available evidence suggests that $20 \%-30 \%$ of the left ventricle needs to be viable to allow improvement in the LVEF.

Besides improvement in the LVEF, improvement in symptoms and exercise capacity may be clinically relevant, although few data are available on these topics. Published studies showed that the mean NYHA class improved significantly in patients with viable myocardium (19-21). Individual data, however, varied significantly, and accurate prediction of improvement in symptoms for an individual patient remains difficult. Aiming at a more objective measure of heart failure symptoms, Marwick et al. $(21,22)$ assessed exercise capacity before and after revascularization. The authors reported that patients with extensive viability showed significant improvement in exercise capacity after revascularization.

Another potential endpoint in viability assessment is the prediction of LV remodeling. LV volumes are powerful predictive parameters, and large trials with angiotensinconverting enzyme inhibitors have shown that reverse LV remodeling is associated with improved survival. Small studies have described the relationship between viability and LV remodeling. Mule et al. (23) reported that patients with residual viability or ischemia (involving $>20 \%$ of the left ventricle) demonstrated reverse remodeling after revascularization, with a significant reduction in both LV endsystolic and end-diastolic volumes after revascularization. These data further support the various benefits of surgery for patients with residual viability. Even more important, patients with predominantly scar tissue exhibited ongoing adverse LV remodeling, with an increase in both LV endsystolic and end-diastolic volumes. Therefore, surgery for patients with scar tissue did not result in reverse LV remodeling.

Although the prevalence of cardiac arrhythmias is substantially elevated in patients with impaired LVEF attributable to chronic coronary artery disease, and internal cardiac defibrillator implantation is now an accepted treatment for these patients, the relationship between viability and ventricular arrhythmias is not clear. Currently, there are no solid data on the prevention of ventricular arrhythmias in patients who have viable myocardium and who are undergoing revascularization.

The final, most important, endpoint is long-term prognosis. A substantial number of studies have evaluated the prognostic value of viability in relation to therapy. These studies consistently showed a low event rate in patients who had viable myocardium and who underwent revascularization, suggesting that revascularization stabilizes the unstable substrate of dysfunctional but viable myocardium. In line with this finding, Rohatgi et al. (24) demonstrated that revascularization in patients with a substantial amount of viable myocardium reduces the number of hospital readmissions for congestive heart failure. Allman et al. (25) performed a meta-analysis of 24 prognostic studies (with 3,088 patients) that used various viability techniques and that showed a $3.2 \%$ annual death rate in patients who had viable myocardium and who underwent revascularization, compared with a $16 \%$ annual death rate in patients who had viable myocardium and who were treated medically (Fig. 1). All of these studies were based on retrospective analyses, and prospective data are needed to determine the precise value of viability in the therapeutic decision-making process. In this regard, randomized clinical trials, such as the Surgical Treatment for Ischemic Heart Failure Trial, are awaited to assess the optimal treatment in these patients.

\section{Viability Techniques}

Many imaging techniques have been proposed over the last 2 decades. These techniques rely on different characteristics of dysfunctional but viable myocardium (Table 3). The most tested and clinically used techniques include nuclear imaging by PET (evaluating glucose use with ${ }^{18} \mathrm{~F}-$ FDG), nuclear imaging by SPECT (evaluating perfusion, cell membrane integrity, and intactness of mitochondria with ${ }^{201} \mathrm{Tl}-$ or ${ }^{99 \mathrm{~m}} \mathrm{Tc}$-labeled agents), echocardiography with dobutamine (to assess contractile reserve), echocardiography with intravenous contrast agents (to assess perfusion), MRI with dobutamine (to assess contractile reserve), MRI with intravenous contrast agents (to assess scar 


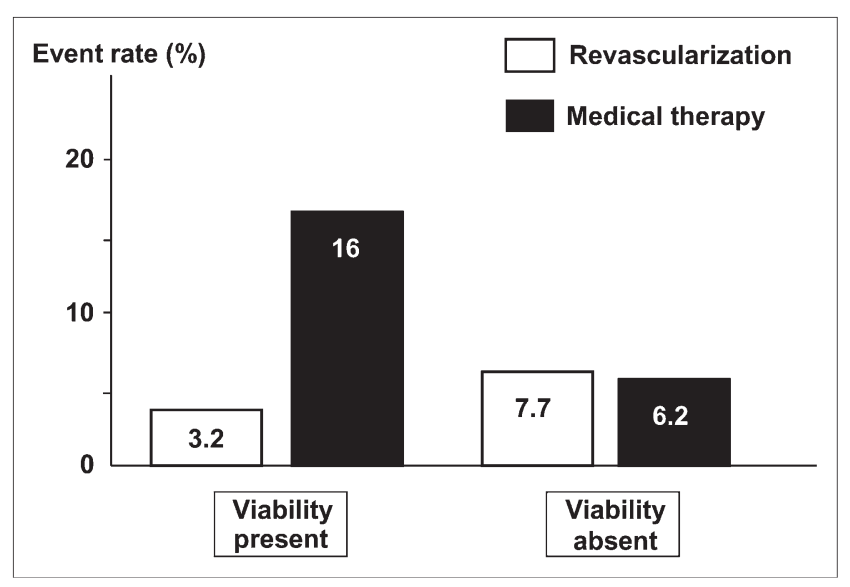

FIGURE 1. Analysis (25) of pooled data from 24 prognostic studies that used different viability techniques and that showed $3.2 \%$ annual death rate in patients who had viable myocardium and who were undergoing revascularization, compared with $16 \%$ annual death rate in patients who had viable myocardium and who were treated medically. Intermediate event rates $(7.7 \%$ and $6.2 \%)$ were observed in patients with nonviable myocardium.

tissue), and CT with intravenous contrast agents (to assess scar tissue). These techniques are discussed in the following text.

\section{Nuclear Imaging by PET with ${ }^{18}$ F-FDG}

Although various tracers have been used in combination with PET $\left({ }^{11} \mathrm{C}\right.$-acetate and $\left.{ }^{82} \mathrm{Rb}\right),{ }^{18} \mathrm{~F}-\mathrm{FDG}$ is the tracer most frequently used to assess myocardial viability. ${ }^{18} \mathrm{~F}$ FDG is used to evaluate cardiac glucose use, and the tracer is a glucose analog (one $\mathrm{OH}$ group is replaced by an ${ }^{18} \mathrm{~F}$ atom). The initial tracer uptake in myocytes is comparable to glucose uptake. After phosphorylation, ${ }^{18} \mathrm{~F}-\mathrm{FDG}-6-\mathrm{PO}_{4}$ remains trapped in myocytes, and further metabolism is not possible, thus providing a strong signal for imaging. Like glucose uptake, cardiac ${ }^{18} \mathrm{~F}-\mathrm{FDG}$ uptake is strongly influenced by metabolic circumstances, in particular, by plasma levels of insulin and free fatty acids. Although insulin stimulates cardiac glucose (and ${ }^{18} \mathrm{~F}-\mathrm{FDG}$ ) uptake, free fatty acids inhibit glucose (and ${ }^{18}$ F-FDG) accumulation. This situation may be mimicked by either oral glucose loading or hyperinsulinemic euglycemic clamping. The majority of cardiac ${ }^{18}$ F-FDG studies have been performed after oral glucose loading, which is a simple and effective approach. The main shortcoming is that image quality for patients with impaired glucose tolerance or overt diabetes is poor. Hyperinsulinemic euglycemic clamping can overcome this problem, but this approach is time-consuming and laborious. An alternative approach may be the use of nicotinic acid derivatives; initial studies have demonstrated adequate image quality, even for patients with diabetes.

Viability Criteria. For the optimal assessment of viability, integration of function, perfusion, and ${ }^{18} \mathrm{~F}-\mathrm{FDG}$ uptake is needed. Regions with contractile dysfunction can exhibit 4 patterns of perfusion and ${ }^{18}$ F-FDG uptake. Viable tissue can display normal perfusion and ${ }^{18} \mathrm{~F}-\mathrm{FDG}$ uptake (chronic stunning) or reduced perfusion and preserved ${ }^{18} \mathrm{~F}-\mathrm{FDG}$ uptake (mismatch pattern, hibernation) (Fig. 2) (26); scar tissue (perfusion- ${ }^{18}$ F-FDG match) can be further divided into subendocardial and transmural scars, depending on the percentage of tracer uptake.

Prediction of Outcome. Twenty studies with ${ }^{18} \mathrm{~F}-\mathrm{FDG}$ PET (total of 598 patients) aimed to predict improvement in regional function after revascularization (18). The mean sensitivity and specificity in these studies were $93 \%$ and $58 \%$, respectively (Table 4) (18). The majority of these studies used combined information on perfusion and ${ }^{18} \mathrm{~F}$ FDG uptake. Moreover, when the studies that used ${ }^{18} \mathrm{~F}-$ FDG alone (without a flow tracer) were excluded from the analysis of pooled data, a sensitivity of $88 \%$ and a specificity of $74 \%$ were obtained. Improvement in global LV function was evaluated in $12{ }^{18} \mathrm{~F}$-FDG PET studies with 333 patients. On average, the LVEF improved from $37 \%$ to $47 \%$ in patients with viable myocardium. In patients without viable myocardium, the LVEF remained unchanged (39\% vs. 40\%) (Table 5) (26).

Two studies $(19,22)$ evaluated the relationship between the presence of viability on ${ }^{18} \mathrm{~F}$-FDG PET before revascularization and improvement in symptoms after revascularization. Both studies indicated that improvement in heart failure symptoms after revascularization occurred predominantly in patients with viable myocardium. Seven ${ }^{18}$ F-FDG PET studies with 619 patients evaluated long-term prognosis in relation to treatment (medical and revascularization) and viability (absent or present) (27-33). Analysis of the pooled data demonstrated that the highest event rate was observed in patients who had viable myocardium and

TABLE 3

Characteristics of Dysfunctional but Viable Myocardium in Relation to Imaging Modalities

\begin{tabular}{ll}
\hline \multicolumn{1}{c}{ Imaging modality } & \multicolumn{1}{c}{ Characteristics of viability } \\
\hline PET or SPECT with ${ }^{18} \mathrm{~F}-\mathrm{FDG}$ & Glucose use \\
SPECT with ${ }^{201} \mathrm{TI}$ & Perfusion and cell membrane integrity \\
SPECT with ${ }^{99 m}$ Tc-labeled tracers & Perfusion, cell membrane integrity, and mitochondrial intactness \\
Contrast echocardiography & Perfusion \\
Echocardiography or MRI with low-dose dobutamine infusion & Contractile reserve \\
Contrast-enhanced MRI & Scar tissue \\
Contrast-enhanced CT & Scar tissue \\
\hline
\end{tabular}




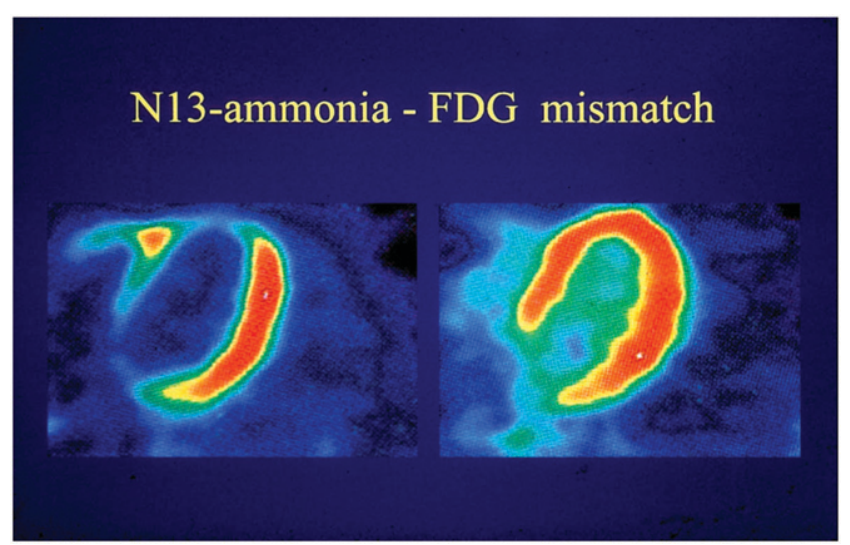

FIGURE 2. PET with ${ }^{13} \mathrm{~N}$-ammonia and ${ }^{18} \mathrm{~F}-\mathrm{FDG}$ to assess myocardial viability (26). Regional myocardial ${ }^{18} \mathrm{~F}-\mathrm{FDG}$ uptake is disproportionately enhanced compared with regional myocardial blood flow; this pattern is termed perfusion-metabolism mismatch and is indicative of hibernating myocardium.

who were treated medically, whereas patients who had viable myocardium and who underwent revascularization had the best prognosis (Table 6).

\section{Nuclear Imaging by SPECT with ${ }^{201} \mathrm{TI}-\mathrm{Chloride}$}

The initial uptake of ${ }^{201} \mathrm{Tl}$ is mainly determined by regional perfusion, whereas sustained uptake, over a longer period of time, depends on cell membrane integrity and thus myocyte viability. Although many protocols are available, the 2 protocols most frequently used are stressredistribution-reinjection imaging and rest-redistribution imaging. The first protocol provides information on both stress-inducible ischemia and viability, whereas the latter provides information only on viability.

Viability Criteria. Four markers of viability are normal ${ }^{201} \mathrm{Tl}$ uptake (normal perfusion) at stress, stress defects with redistribution (reversible defects) on 3- to 4-h delayed images, redistribution in fixed defects on images at redistribution after reinjection or on delayed rest images (Fig. 3) (frequently a threshold of a $10 \%$ increase in tracer uptake is used), and tracer uptake of greater than $50 \%$ on redistributionreinjection images or on delayed rest images. The first 3 markers reflect jeopardized but viable myocardium, but the fourth marker is more complex. Frequently, segments with tracer uptake of greater than $50 \%$ do not improve in func-
TABLE 4

Pooled Data from Viability Studies of Bax et al. (18) with Different Techniques to Predict Improvement in LVEF After Revascularization

\begin{tabular}{lccccc}
\hline & No. of & $\%$ & $\%$ & $\%$ & $\%$ \\
Technique & studies & Sensitivity & $\begin{array}{c}\% \\
\text { Specificity }\end{array}$ & $\begin{array}{c}\% \\
\text { NPV }\end{array}$ & PPV \\
\hline${ }^{18}$ F-FDG PET & 20 & 93 & 58 & 85 & 77 \\
${ }^{201}$ TI imaging & 33 & 87 & 55 & 81 & 64 \\
99mTc-labeled & 20 & 81 & 66 & 77 & 71 \\
tracers & & & & & \\
DSE & 32 & 81 & 80 & 85 & 77
\end{tabular}

DSE = dobutamine stress echocardiography; NPV = negative predictive value; PPV = positive predictive value.

tion; the reason for this observation is the presence of nontransmural infarction, rather than jeopardized, hibernating myocardium (assuming adequate revascularization). Segments with nontransmural infarction contain viable tissue (and thus frequently exhibit $>50 \%$ tracer uptake) but are not always capable of showing improvement in function after revascularization because of the presence of fibrosis.

Prediction of Outcome. Thirty-three studies (22 with rest-redistribution and 11 with a reinjection protocol) (total of 858 patients) focused on the prediction of improvement in regional function after revascularization (18). The mean sensitivity and specificity in these studies were $86 \%$ and $59 \%$, respectively (Table 4) (18). The lower specificity may be related to the definition of viable myocardium; as stated earlier, segments with tracer uptake of greater than 50\% are classified as viable but frequently contain subendocardial scar tissue and may not improve in function after revascularization. A higher accuracy for the prediction of improvement in function was obtained when inducible ischemia was present in segments with tracer uptake of greater than $50 \%(34)$.

Improvement in global LV function was evaluated in 5 studies with 96 patients; on average, the LVEF improved from $30 \%$ to $38 \%$ in patients with viable myocardium. In patients without viable myocardium, the LVEF remained unchanged (29\% vs. $31 \%$ ) (Table 5).

Only one study focused on the prediction of improvement in heart failure symptoms; Mule et al. (23) demonstrated that

TABLE 5

Pooled Data from Viability Studies of Maddahi et al. (26) with Different Techniques to Predict Improvement in LVEF After Revascularization

\begin{tabular}{|c|c|c|c|c|c|}
\hline \multirow[b]{2}{*}{ Technique } & \multirow[b]{2}{*}{ No. of studies (no. of patients) } & \multicolumn{2}{|c|}{ Viability present } & \multicolumn{2}{|c|}{ Viability absent } \\
\hline & & \% LVEF pre & $\%$ LVEF post & $\%$ LVEF pre & $\%$ LVEF post \\
\hline${ }^{18}$ F-FDG PET & $12(333)$ & 37 & 47 & 39 & 40 \\
\hline $201 \mathrm{TI}$ & $5(96)$ & 30 & 38 & 29 & 31 \\
\hline 99mTc-labeled tracers & $4(75)$ & 47 & 53 & 40 & 39 \\
\hline DSE & $8(254)$ & 35 & 43 & 35 & 36 \\
\hline
\end{tabular}


TABLE 6

Relationship Among Myocardial Viability, Type of Treatment, and Event Rates in Patients with LV Dysfunction and Chronic Coronary Artery Disease*

\begin{tabular}{|c|c|c|c|c|c|}
\hline \multirow[b]{3}{*}{ Technique } & \multirow[b]{3}{*}{$\begin{array}{l}\text { No. of studies } \\
\text { (no. of patients) }\end{array}$} & \multicolumn{4}{|c|}{$\%$ Event rate } \\
\hline & & \multicolumn{2}{|c|}{ Viability present } & \multicolumn{2}{|c|}{ Viability absent } \\
\hline & & Revascularization & $\begin{array}{c}\text { Pharmacologic } \\
\text { treatment }\end{array}$ & Revascularization & $\begin{array}{c}\text { Pharmacologic } \\
\text { treatment }\end{array}$ \\
\hline${ }^{18} \mathrm{~F}-F D G$ PET & 7 (619) & 7 & 29 & 12 & 12 \\
\hline DSE & $6(686)$ & 6 & 22 & 16 & 28 \\
\hline
\end{tabular}

patients with jeopardized myocardium showed significant improvement in the NYHA class after revascularization. Long-term prognosis for patients was evaluated by ${ }^{201} \mathrm{Tl}$ imaging (35-38); these studies were uniform in demonstrating that superior long-term survival was present in patients who had viable myocardium and who underwent revascularization. For example, Pagley et al. (36) studied 70 patients with multivessel disease and depressed LVEF, and all underwent surgical revascularization. The cardiac death rate was significantly lower in patients with viable myocardium than in patients without viable myocardium $(18 \%$ vs. $41 \% ; P<0.05)$.

\section{Nuclear Imaging by SPECT with ${ }^{99 m}$ Tc-Labeled Agents}

The uptake and retention of sestamibi are dependent on perfusion, cell membrane integrity, and mitochondrial function (membrane potential) and thus are markers of viable tissue.

Viability Criteria. The most commonly used viability criterion is the percentage of tracer uptake in dysfunctional segments. As in ${ }^{201} \mathrm{Tl}$ imaging, a $50 \%-60 \%$ cutoff value is frequently used. Schneider et al. (39) pointed out that it may be preferable to use uptake cutoff values for the inferior or septal segments that are different from those used for other regions, because attenuation is important in these segments and thus tracer uptake will always be lower.

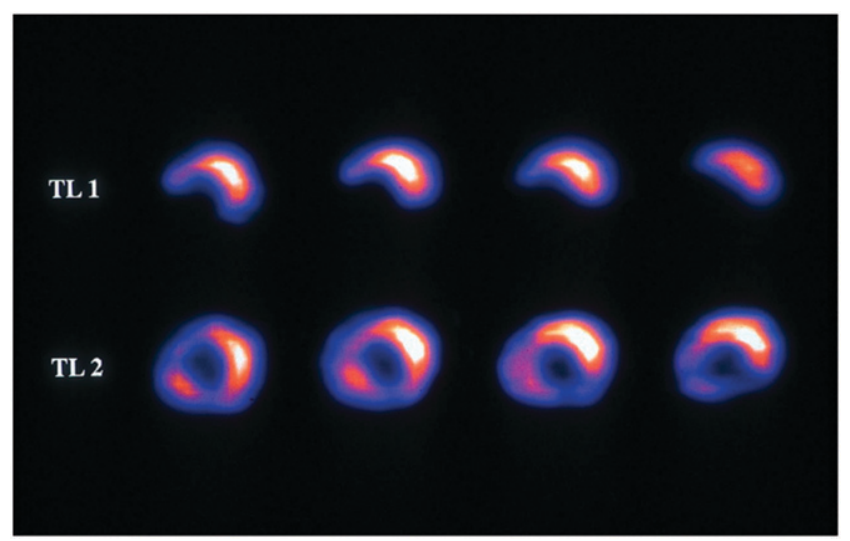

FIGURE 3. Corresponding series of ${ }^{201} \mathrm{TI}$ rest-redistribution SPECT short-axis slices. Early slices (top) show defect in inferoseptal wall, with redistribution on late slices (bottom).
Sestamibi SPECT has been performed after the administration of nitrates in several studies. It is thought that nitrates enhance blood flow (and thus tracer uptake) to myocardial regions that are subtended by severely stenosed arteries. In most nitrate-enhanced studies, 2 sets of images are obtained: a resting image and a nitrate-enhanced image; defect reversibility after nitrate administration (i.e., defect filling in) is considered to be indicative of viability.

Prediction of Outcome. Twenty studies (7 after nitrate administration) (total of 488 patients) focused on the prediction of improvement in regional function after revascularization (18). The mean sensitivity and specificity in these studies were $81 \%$ and $66 \%$, respectively (Table 4) (18). The majority of these studies used a resting image, and segments were classified as viable when activity exceeded a certain threshold (frequently 50\%-60\%). The use of thresholds (as in ${ }^{201} \mathrm{Tl}$ studies) may result in lower specificity because the identification of nontransmural infarction is not possible.

Accordingly, nitrate-enhanced studies should have a higher accuracy; indeed, when nitrate-enhanced studies were analyzed separately (7,180 patients), a sensitivity of $86 \%$ and a specificity of $83 \%$ were obtained. Improvement in the LVEF was evaluated in 4 studies with 75 patients. The LVEF improved from $47 \%$ to $53 \%$ in patients with viable myocardium, whereas the LVEF did not change in patients without viable myocardium (40\% vs. 39\%) (Table 5). No studies focusing on the prediction of improvement in heart failure symptoms in relation to viability have been done with sestamibi imaging. Thus far, one study in which sestamibi imaging was used to evaluate long-term prognosis for patients with ischemic cardiomyopathy has been published (40). Sciagra et al. (40) evaluated 105 patients with chronic coronary artery disease and LV dysfunction; all underwent nitrate-enhanced sestamibi imaging. The patients were accordingly divided into 3 groups: medical treatment, complete revascularization, and incomplete revascularization. Superior survival was shown for the patients with complete revascularization compared with the other 2 groups. The most important prognostic predictor of future cardiac events was the number of nonrevascularized dysfunctional regions with viable tissue on sestamibi imaging. 
Therefore, these data also indicate the poor prognosis for patients who have viable myocardium but who do not receive adequate revascularization.

\section{Echocardiography with Low-Dose Dobutamine}

Over the years, echocardiography has been used extensively for the assessment of myocardial viability. The simple assessment of LV end-diastolic wall thickness can already be used to obtain a first impression of the presence or absence of viable tissue. It has been demonstrated that severely thinned walls most likely represent scar tissue. In a study of a large registry, Schinkel et al. demonstrated that segments with LV end-diastolic wall thicknesses of less than $6 \mathrm{~mm}$ virtually never exhibited contractile reserve (41). On the other hand, the majority of segments with relatively well preserved end-diastolic wall thicknesses ( $\geq 6 \mathrm{~mm}$ ) had contractile reserve. Low-dose dobutamine echocardiography has been used to further refine viability assessment.

Viability Criteria. Infusions of low-dose dobutamine (5-10 $\mu \mathrm{g} / \mathrm{kg} / \mathrm{min}$ ) have been demonstrated to increase contractility (without a substantial increase in heart rate) in dysfunctional but viable myocardium; this property has been referred to as contractile reserve. Segments without viable myocardium do not show this contractile reserve. In recent years, the protocol has been extended to high-dose dobutamine infusion, which allows the assessment of ischemia (18). With this protocol (with infusions of up to $40 \mu \mathrm{g} / \mathrm{kg} / \mathrm{min}$, with the addition of atropine if needed), 4 response patterns can be observed: biphasic response (initial improvement followed by worsening of wall motion), worsening (direct deterioration of wall motion without initial improvement), sustained improvement (improvement of wall motion without subsequent deterioration), and no change (no change in wall motion during the entire study). The first pattern most likely represents viability with superimposed ischemia, whereas the second pattern represents severe ischemia in a region subtended by a critically stenosed artery. The third pattern is probably related to subendocardial necrosis, whereas the fourth pattern represents a transmural scar.

Prediction of Outcome. Thirty-two studies used dobutamine echocardiography (total of 1,090 patients) to predict improvement in regional function after revascularization (18). The mean sensitivity and specificity were $82 \%$ and $79 \%$, respectively (Table 4) (18). Most of these studies used low-dose dobutamine echocardiography, whereas 4 studies used a low-high dose protocol (18). Improvement in global LV function was evaluated in 7 studies with 254 patients. On average, the LVEF improved from $35 \%$ to $43 \%$ in patients with viable myocardium. In patients without viable myocardium, the LVEF remained unchanged (35\% vs. $36 \%$ ) (Table 5). Two studies evaluated functional status before and after revascularization in relation to the absence or presence of viable myocardium $(21,42)$. It was demonstrated that the majority of patients with viable myocar- dium showed improvement in the NYHA class after revascularization.

Six studies with 686 patients evaluated long-term prognosis in relation to treatment (medical and revascularization) and viability (absent or present) (42-47). The patients were divided into 4 groups similar to those in the ${ }^{18} \mathrm{~F}-\mathrm{FDG}$ PET studies described earlier; analysis of the pooled data demonstrated that the lowest event rate $(6 \%)$ was observed in patients who had viable myocardium and who underwent revascularization, whereas the event rates in the other 3 groups were similar (Table 6).

\section{Echocardiography with Intravenous Contrast Agents}

The improved technical properties of myocardial contrast agents now allow for the assessment of myocardial perfusion. In early studies, intracoronary injection of contrast agents was still needed, but with the newer generation of contrast agents, intravenous administration is possible. The newer contrast agents are composed of high-molecularweight inert gases. The microbubbles stay in the vascular space and do not enter the extravascular space; within the vascular space, microbubbles behave like red cells in terms of rheology and can be used in combination with echocardiography to visualize myocardial perfusion directly.

Viability Criteria. Because myocardial perfusion is a prerequisite for myocardial viability, myocardial contrast echocardiography has been used to assess myocardial viability. It has been shown that the contrast echocardiography parameters of myocardial perfusion correlate positively with microvascular density and capillary area and inversely with the extent of fibrosis (48). In the clinical setting, myocardial perfusion is evaluated qualitatively by myocardial contrast echocardiography, and segments are visually classified as being viable, with normal or patchy perfusion, and nonviable when perfusion is absent.

Prediction of Outcome. Not many studies in which contrast echocardiography was used for the prediction of functional recovery have been published. A small direct comparison of dobutamine stress echocardiography, ${ }^{201} \mathrm{Tl}$ imaging, and contrast echocardiography was performed with 18 patients undergoing revascularization (49). Both ${ }^{201} \mathrm{Tl}$ imaging and contrast echocardiography had a high sensitivity and a relatively low specificity for the prediction of improvement in regional function after revascularization, whereas dobutamine stress echocardiography had a lower sensitivity and a relatively high specificity. In that particular study, contrast echocardiography had a sensitivity of $89 \%$ and a specificity of $51 \%$. Two other studies with contrast echocardiography confirmed this observation and demonstrated a high sensitivity and a lower specificity $(50,51)$. The lower specificity was related to the same problem as with ${ }^{201} \mathrm{Tl}$ scintigraphy and was related to the inability to identify subendocardial scar tissue, resulting in an overprediction of recovery of function. One study demonstrated that patients with 3 or more viable segments on contrast echocardiography had a high likelihood of showing 
improvement in global LV function after revascularization (49). Currently, no studies evaluating improvement in symptoms or long-term outcome with contrast echocardiography are available.

\section{MRI}

MRI is a relatively new technique for the detection of viability. Resting LV dysfunction can be precisely assessed by use of cine acquisitions with tissue tagging and strain analysis (52). As with echocardiography, the assessment of LV enddiastolic wall thickness is useful for ruling out viability. In addition, contractile reserve during the infusion of low-dose dobutamine can also be assessed by MRI. Finally, infarcted myocardium can be visualized with substantially improved image quality compared with the quality of traditional spinecho images by use of an optimized segmented inversion recovery gradient-recalled echo sequence (53).

Viability Criteria. Various studies have used an LV enddiastolic wall thickness of less than $5.5 \mathrm{~mm}$ as a marker of scar tissue. Baer et al. (54) compared LV end-diastolic wall thickness on MRI with glucose use on ${ }^{18}$ F-FDG PET and demonstrated that regions with an end-diastolic wall thickness of less than $5.5 \mathrm{~mm}$ had reduced glucose use, whereas regions with an end-diastolic wall thickness of $\geq 5.5 \mathrm{~mm}$ had preserved glucose use.

The evaluation of contractile reserve by MRI is similar to that with echocardiography, but the available MRI studies have used only low-dose dobutamine infusion.

Contrast hyperenhancement on delayed rest MRI is defined as regions with increased intensity on T1-weighted images acquired more than $5 \mathrm{~min}$ after the intravenous administration of a contrast agent. The mechanism underlying the hyperenhancement is related to the interstitial space between collagen fibers, which is larger in scar tissue than in normal myocardium, and the contrast agent is trapped in these infarcted regions. In animal experiments, excellent agreement between the extent of hyperenhancement on contrast-enhanced MRI and the histologically determined infarct size was demonstrated (55). The major advantage of contrast-enhanced MRI is superior spatial resolution, which allows differentiation between transmural necrosis and subendocardial necrosis.

Prediction of Outcome. Segments with an end-diastolic wall thickness of less than $5.5 \mathrm{~mm}$ virtually never show recovery of function after revascularization. On the contrary, segments with a wall thickness of $\geq 5.5 \mathrm{~mm}$ do not always show improvement in function after revascularization, a finding that may be related to the presence of nontransmural infarction. Segments with an end-diastolic wall thickness of $\geq 5.5 \mathrm{~mm}$ frequently contain subendocardial scar tissue, with residual viability in the epicardial layers (Fig. 4). However, without jeopardized myocardium being present, recovery of function will not occur after revascularization. Three studies with 100 patients used wall thickness to predict functional recovery, and pooling of the data revealed a sensitivity of $95 \%$ and a specificity of $41 \%$ (56). Accordingly, severe wall
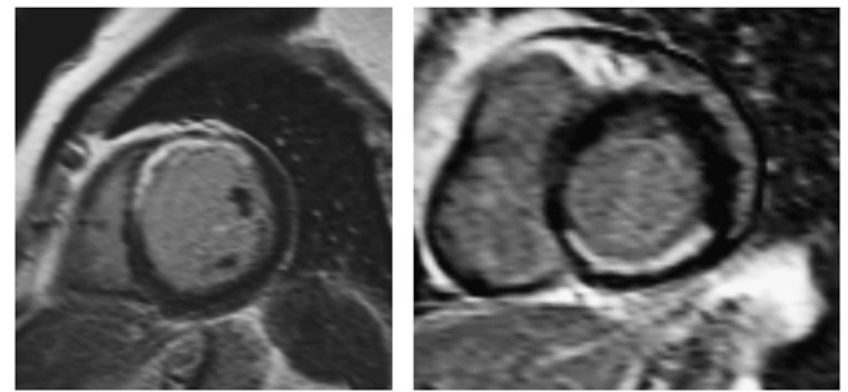

FIGURE 4. Contrast-enhanced MRI studies. (Left) Short-axis slice from patient with (not completely transmural) anteroseptal infarct. (Right) Short-axis slice from patient with subendocardial inferior infarct.

thinning suggests scar tissue, with a high accuracy for predicting no recovery after revascularization. It should be noted, however, that even in the presence of severe wall thinning, recovery of function may occur, but only when contrast-enhanced MRI excludes scar tissue.

Baer et al. (54) extensively explored dobutamine MRI and showed that an increased systolic wall thickening of greater than $2 \mathrm{~mm}$ during dobutamine infusion was an accurate predictor of functional recovery. Bove et al. (57) showed that in segments with limited scar tissue, a normal dobutamine response helps to differentiate segments with greater functional recovery after revascularization. Nine studies with 252 patients evaluated the role of dobutamine MRI and found a mean sensitivity of $73 \%$ (range, $50 \%-89 \%$ ) and a mean specificity of $83 \%$ (range, $70 \%-95 \%$ ) (56).

Studies with contrast-enhanced MRI showed that the likelihood of recovery of function after revascularization paralleled the transmural nature of the infarction. The amount of hyperenhancement is a very good indicator of improvement in function: improvement in function decreases progressively as the transmural nature of scar tissue increases $(58,59)$. Selvanayagam et al. (60) demonstrated that late-enhancement MRI is a powerful predictor of myocardial viability after surgery, suggesting an important role for this technique in clinical viability assessment. Moreover, MRI may be particularly useful for evaluating impaired resting myocardial blood flow in hibernating regions beyond a coronary stenosis, myocardial scarring resulting from revascularization procedures, and improvement in regional myocardial contractility after surgical ventricular reconstruction (60-62). Pooling of the data from the 4 studies with contrast-enhanced MRI for 132 patients undergoing revascularization revealed a sensitivity of $95 \%$ and a specificity of $45 \%$ (56). The suboptimal specificity was again related to the presence of segments with subendocardial necrosis (and epicardial viability), which do not show improvement in function.

\section{CT}

Rapid technical developments in CT of the heart, including fast ECG-synchronization, multirow detector acquisition, 
and optimization of scan protocols, have led to the increased clinical use of this technique for noninvasive coronary angiography. Some studies have evaluated CT for the assessment of myocardial viability, although the latter is not the current primary use of cardiac CT.

Viability Criteria. LV end-diastolic wall thickness can be used as a marker of scar tissue. Additionally, contrast hyperenhancement on delayed rest CT is related to myocardial infarction. In the setting of reperfusion after acute myocardial infarction, Mahnken et al. (63) studied 28 patients with contrast-enhanced cardiac CT and late-enhancement MRI. Contrast enhancement patterns demonstrated good agreement between late-enhancement MRI and late-enhancement CT. Initial experience by Nikolaou et al. (64) with contrastenhanced CT for 30 patients late after myocardial infarction showed a systematic underestimation of the true infarct size compared with the results of late-enhancement MRI. It is clear that further studies on the role of CT in viability assessment are needed; the idea of evaluating coronary anatomy and myocardial viability with a single technique is appealing, although radiation exposure may be a limiting factor.

\section{Nuclear Imaging Versus Dobutamine Stress Echocardiography}

A controversial issue in clinical cardiology concerns the relative merits of nuclear imaging and dobutamine echocardiography for the assessment of viability. Various direct comparisons of nuclear imaging and low-dose dobutamine stress echocardiography have reported substantial disagreement between the techniques. For example, Panza et al. (65) reported an agreement between ${ }^{201} \mathrm{Tl}$ imaging and lowdose dobutamine echocardiography of only $68 \%$. The disagreement was mainly related to segments with viability on nuclear imaging but without contractile reserve on echocardiography. The largest head-to-head comparison included 114 patients who had ischemic cardiomyopathy and who underwent resting perfusion imaging (with ${ }^{99 \mathrm{~m}} \mathrm{Tc}$-tetrofosmin) and low-dose dobutamine echocardiography (66). The agreement between the techniques was $72 \%$; $92 \%$ of segments without perfusion did not have contractile reserve, but $47 \%$ of segments with perfusion also lacked contractile reserve. Accordingly, the available studies showed a higher sensitivity of nuclear imaging than of dobutamine echocardiography for detecting myocardial viability.

Prediction of Outcome. Perrone-Filardi et al. (67) performed a direct comparison of low-dose dobutamine echocardiography and ${ }^{201} \mathrm{Tl}$ rest-redistribution imaging in 40 patients referred for revascularization. The authors reported a significantly higher sensitivity for ${ }^{201} \mathrm{Tl}$ imaging than for low-dose dobutamine echocardiography (100\% vs. $79 \%$; $P<0.05)$. The specificities of the techniques were comparable (78\% vs. 79\%). When data from all available studies with a direct comparison of nuclear imaging and low-dose dobutamine echocardiography were pooled, the higher sensitivity for nuclear imaging was confirmed. In particular, data from 11 studies with 325 patients showed sensitivities of $90 \%$ for nuclear imaging and $74 \%$ for dobutamine echocardiography $(P<0.05)(18)$. Conversely, the specificity of low-dose dobutamine echocardiography was higher (57\% vs. 78\%; $P<$ 0.05 ). Accordingly, the integration of perfusion (a very sensitive marker) and contractile reserve (a very specific marker) may further improve the prediction of functional recovery.

\section{Temporal Relationship Among Viability, Revascularization, and Functional Outcome}

It has been demonstrated that once viability has been detected, revascularization should not be delayed. Beanlands et al. (68) studied 35 patients with ${ }^{18} \mathrm{~F}$-FDG PET; patients were divided into 2 groups based on the median waiting time after PET: an early group $(<35 \mathrm{~d} ; n=18)$ and a late group ( $\geq 35 \mathrm{~d} ; n=17$ ). In the early revascularization group, the LVEF improved after surgery, whereas the patients with late revascularization did not show improvement in the LVEF. Bax et al. (69) studied 85 patients with dobutamine stress echocardiography; all had substantial viability. Forty patients underwent early revascularization $(20 \pm 12$ [mean \pm SD] d after stress echocardiography), and 45 underwent late revascularization $(85 \pm 47 \mathrm{~d}$ after stress echocardiography). In the early revascularization group, the LVEF improved significantly during follow-up, whereas the patients with late revascularization did not show improvement in the LVEF (Fig. 5). Moreover, the 2-y mortality rates were $5 \%$ in the early group and $20 \%$ in the late group $(P<0.05)$. These observations support the clinical relevance of assessment of viability in patients with ischemic cardiomyopathy.

\section{Additional Information Needed Besides Myocardial Viability}

Surgery for patients with heart failure has expanded from revascularization only to combined approaches involving

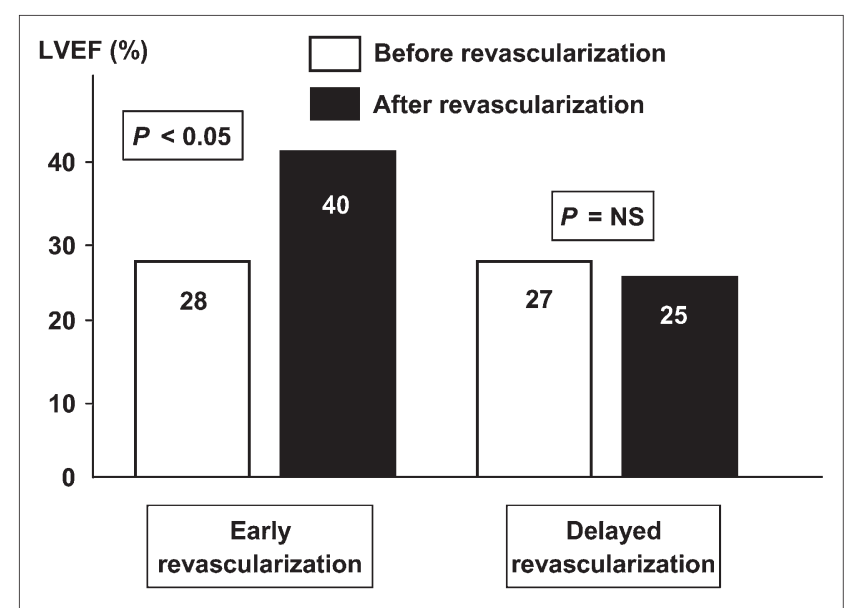

FIGURE 5. Effect of delayed vs. timely revascularization on change in LVEF in patients with substantial viability on dobutamine stress echocardiography (69). Patients with early revascularization showed significant improvement in LVEF after revascularization, which was not observed after delayed revascularization. 
mitral valve repair, LV aneurysmectomy, or LV restoration. Accordingly, more information is needed before surgery to help cardiac surgeons, anesthetists, and cardiologists determine the optimal surgical technique.

Obviously, information about the LVEF is needed to assess the risk of surgery. It is well known that patients with a low LVEF are at higher risk for (peri)operative morbidity and mortality. Nevertheless, because of advances in surgical techniques and optimization of perioperative metabolic and mechanical support, coronary artery bypass surgery has become realistic even in patients with the most severe LV dysfunction.

The evaluation of LV volumes is relevant for determination of the likelihood of recovery of function after revascularization. It was recently demonstrated that patients with severely dilated left ventricles have a low likelihood of showing improvement in the LVEF despite the presence of substantial viability (70). The change in the LVEF after revascularization was linearly related to the baseline LV end-systolic volume, with a higher end-systolic volume being associated with a low likelihood of functional recovery after revascularization. Patients with substantial viability ( $\geq 4$ viable segments) and LV end-systolic volumes of less than $130 \mathrm{~mL}$ had the best 3-year survival rates, whereas patients without substantial viability and LV end-systolic volumes of greater than $130 \mathrm{~mL}$ had the worst survival rates (Fig. 6) (70).

Information on the extent of scar tissue may assist in the prediction of recovery of global LV function in patients with ischemic cardiomyopathy (71). The PARR-1 study (71) proposed a prediction model that combined PET and clinical parameters to estimate the likelihood of recovery of LV function after surgery; in this model, the amount of scar tissue was the most important predictor. In patients with extensive scar tissue, bypass surgery could be combined

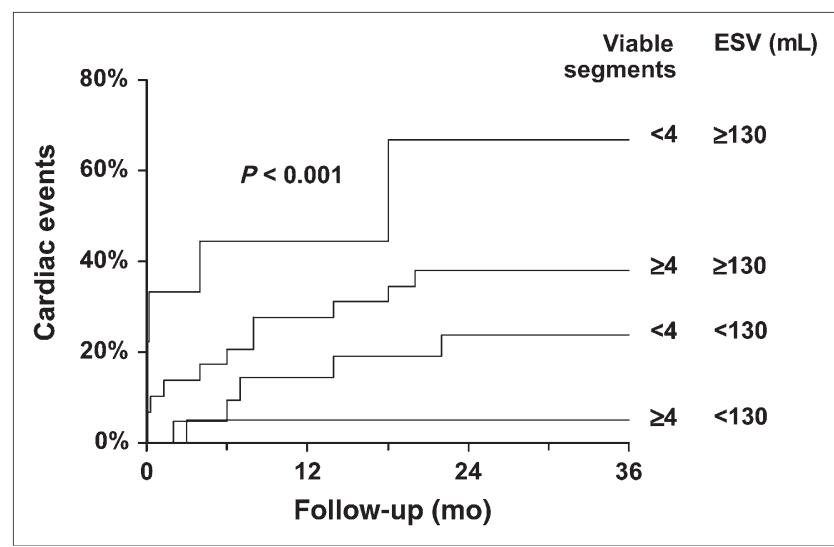

FIGURE 6. Cardiac events (including cardiac death, infarction, and hospitalization for heart failure) during 3-y follow-up according to substantial viability ( $\geq 4$ viable segments) and LV end-systolic volume (ESV). Patients with small LV (ESV of $<130$ $\mathrm{mL}$ ) and substantial viability had best prognosis (5\% event rate), whereas patients without viability and large LV (ESV of $\geq 130$ $\mathrm{mL}$ ) had worst prognosis (67\% event rate). (Modified from (70).) with resection of nonviable scar tissue. Several studies have demonstrated that surgical reduction of dyskinetic (cardiac aneurysms) and akinetic regions may reduce wall stress and improve geometry and LV function in some patients. In selected patients with a previous anterior myocardial infarction, surgical anterior ventricular endocardial restoration is a safe and effective surgical procedure for restoring geometry and reverse LV remodeling. With the increasing use of LV aneurysmectomy, information on the presence, location, and extent of LV aneurysms is needed and can be provided by contrast-enhanced MRI.

Outcome after surgery may also be influenced by the presence of ischemic mitral valve regurgitation. This phenomenon may occur as a consequence of mitral valve annular dilatation, and in the presence of significant mitral valve regurgitation, mitral valve repair should be considered in addition to revascularization. Currently, optimal information on the presence, severity, and mechanism of mitral valve regurgitation is obtained from transesophageal echocardiography. Ischemic mitral valve regurgitation can also be visualized adequately by MRI (Fig. 7). Further studies are needed to determine the value of these additional surgical procedures in patients with a substantial amount of dysfunctional but viable myocardium.

\section{CONCLUSIONS AND FUTURE DIRECTIONS}

The assessment of myocardial viability has become an integrated part of the diagnostic and prognostic work-up of patients with heart failure symptoms attributable to ischemic cardiomyopathy. The available evidence suggests that patients with substantial viability will show improvement in function and symptoms after revascularization. In addition, reverse remodeling may occur, and long-term prognosis is

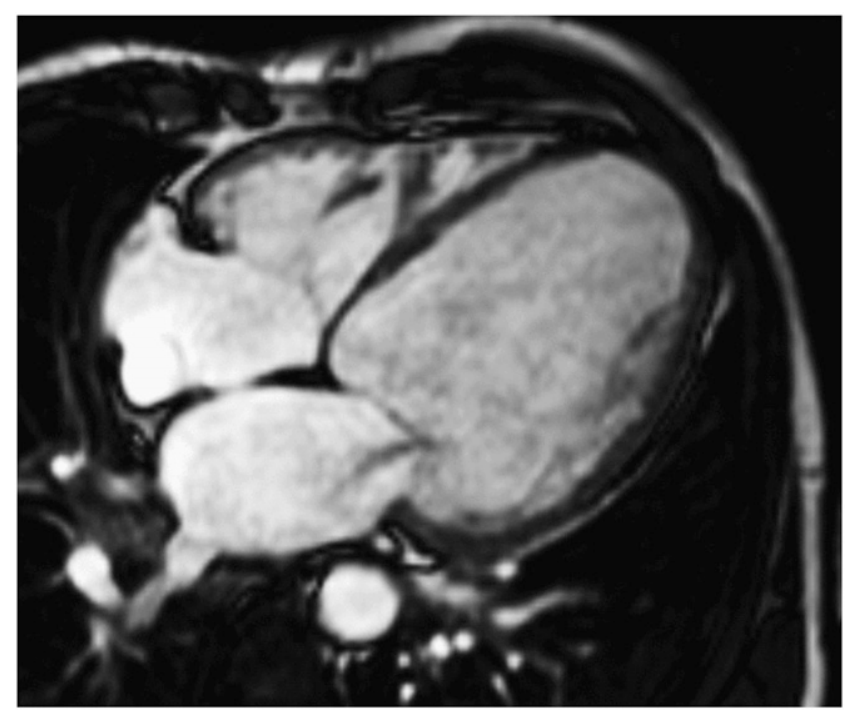

FIGURE 7. Contrast-enhanced MRI study. Four-chamber view of heart of patient with ischemic cardiomyopathy and LV dilatation; note (ischemic) mitral valve regurgitation secondary to annular dilatation. 
good. In contrast, patients without viability will not benefit from revascularization, and the high risk of surgery should be avoided.

Prospective, randomized trials are needed to confirm these findings. It is anticipated that the Surgical Treatment for Ischemic Heart Failure Trial may provide the final evidence on the beneficial effects of the assessment of myocardial viability in combination with surgical revascularization.

\section{REFERENCES}

1. American Heart Association. Heart Disease and Stroke Statistics, 2006 Update. American Heart Association; 2006.

2. Gheorghiade M, Bonow RO. Chronic heart failure in the United States: a manifestation of coronary artery disease. Circulation. 1998;97:282-289.

3. Levy D, Kenchaiah S, Larson MG, et al. Long-term trends in the incidence of and survival with heart failure. N Engl J Med. 2002;347:1397-1402.

4. Schinkel AFL, Poldermans D, Vanoverschelde JLJ, et al. Incidence of recovery of contractile function following revascularization in patients with ischemic left ventricular dysfunction. Am J Cardiol. 2004;93:14-17.

5. Al-Mohammad A, Mahy IR, Norton MY, et al. Prevalence of hibernating myocardium in patients with severely impaired ischaemic left ventricles. Heart. 1998;80:559-564.

6. Auerbach MA, Schöder H, Gambhir SS, et al. Prevalence of myocardial viability as detected by positron emission tomography in patients with ischemic cardiomyopathy. Circulation. 1999;99:2921-2926.

7. Schinkel AFL, Bax JJ, Sozzi FB, et al. Prevalence of myocardial viability assessed by single photon emission computed tomography in patients with chronic ischaemic left ventricular dysfunction. Heart. 2002;88:125-130.

8. Fox KF, Cowie MR, Wood DA, et al. Coronary artery disease as the cause of incident heart failure in the population. Eur Heart J. 2001;22:228-236.

9. Schinkel AFL, Bax JJ, Boersma E, Elhendy A, Roelandt JRTC, Poldermans D. How many patients with ischemic cardiomyopathy exhibit viable myocardium? Am J Cardiol. 2001;88:561-564.

10. Cleland JG, Pennell DJ, Ray SG, et al. Myocardial viability as a determinant of the ejection fraction response to carvedilol in patients with heart failure (CHRISTMAS trial): randomised controlled trial. Lancet. 2003;362:14-21.

11. Rahimtoola SH. The hibernating myocardium. Am Heart J. 1989;117:211-221.

12. Heyndrickx GR, Millard RW, McRitchie RJ, Maroko PR, Vatner F. Regional myocardial functional and electrophysiological alterations after brief coronary artery occlusion in conscious dogs. J Clin Invest. 1975;56:978-985.

13. Braunwald E, Kloner RA. The stunned myocardium: prolonged, postischemic ventricular dysfunction. Circulation. 1982;66:1146-1149.

14. Vanoverschelde JLJ, Wijns W, Depre C, et al. Mechanisms of chronic regional postischemic dysfunction in humans: new insights from the study of noninfarcted collateral-dependent myocardium. Circulation. 1993;87:1513-1523.

15. Elsasser A, Muller KD, Skwara W, Bode C, Kubler W, Vogt AM. Severe energy deprivation of human hibernating myocardium as possible common pathomechanism of contractile dysfunction, structural degeneration and cell death. J Am Coll Cardiol. 2002;39:1189-1198.

16. Depre C, Vanoverschelde J, Gerber B, et al. Correlation of functional recovery with myocardial blood flow, glucose uptake, and morphologic features in patients with chronic left ventricular ischemic dysfunction undergoing coronary artery bypass grafting. J Thorac Cardiovasc Surg. 1997;113:371-378.

17. Bonow RO. Contractile reserve and coronary blood flow reserve in collateraldependent myocardium. J Am Coll Cardiol. 1999;33:705-707.

18. Bax JJ, Poldermans D, Elhendy A, Boersma E, Rahimtoola SH. Sensitivity, specificity, and predictive accuracies of various noninvasive techniques for detecting hibernating myocardium. Curr Probl Cardiol. 2001;26:142-186.

19. DiCarli MF, Asgarzadie F, Schelbert HR, et al. Quantitative relation between myocardial viability and improvement in heart failure symptoms after revascularization in patients with ischemic cardiomyopathy. Circulation. 1995;92:3436-3444.

20. Bax JJ, Visser FC, Poldermans D, et al. Relationship between preoperative viability and postoperative improvement in LVEF and heart failure symptoms. J Nucl Med. 2001;42:79-86.

21. Marwick TH, Zuchowski C, Lauer MS, Secknus MA, Williams MJ, Lytle BW. Functional status and quality of life in patients with heart failure undergoing coronary bypass surgery after assessment of myocardial viability. $\mathrm{J}$ Am Coll Cardiol. 1999;33:750-758.
22. Marwick TH, Nemec JJ, Lafont A, Salcedo EE, MacIntyre WJ. Prediction by postexercise fluoro-18 deoxyglucose positron emission tomography of improvement in exercise capacity after revascularization. Am J Cardiol. 1992;69:854-859.

23. Mule J, Bax JJ, Zingone B, et al. The beneficial effect of revascularization on jeopardized myocardium: reverse remodeling and improved long-term prognosis. Eur J Cardiothorac Surg. 2002;22:426-430.

24. Rohatgi R, Epstein S, Henriquez J, et al. Utility of positron emission tomography in predicting cardiac events and survival in patients with coronary artery disease and severe left ventricular dysfunction. Am J Cardiol. 2001;87:1096-1099.

25. Allman KC, Shaw LJ, Hachamovitch R, Udelson JE. Myocardial viability testing and impact of revascularization on prognosis in patients with coronary artery disease and left ventricular dysfunction: a meta-analysis. J Am Coll Cardiol. 2002;39:1151-1158.

26. Maddahi J, Schelbert H, Brunken R, Di Carli M. Role of thallium-201 and PET imaging in evaluation of myocardial viability and management of patients with coronary artery disease and left ventricular dysfunction. J Nucl Med. 1994;35: 707-715.

27. Di Carli M, Davidson M, Little R, et al. Value of metabolic imaging with positron emission tomography for evaluating prognosis in patients with coronary artery disease and left ventricular dysfunction. Am J Cardiol. 1994;73:527-533.

28. Eitzman D, Al-Aouar ZR, Kanter HL, et al. Clinical outcome of patients with advanced coronary artery disease after viability studies with positron emission tomography. J Am Coll Cardiol. 1992;20:559-565.

29. vom Dahl J, Altehoefer C, Sheehan FH, et al. Effect of myocardial viability assessed by technetium-99m-sestamibi SPECT and fluorine-18-FDG PET on clinical outcome in coronary artery disease. J Nucl Med. 1997;38:742-748.

30. Yoshida K, Gould KL. Quantitative relation of myocardial infarct size and myocardial viability by positron emission tomography to left ventricular ejection fraction and 3-year mortality with and without revascularization. J Am Coll Cardiol. 1993;22:984-997.

31. Lee KS, Marwick TH, Cook SA, et al. Prognosis of patients with left ventricular dysfunction, with and without viable myocardium after myocardial infarction: relative efficacy of medical therapy and revascularization. Circulation. 1994;90: 2687-2694.

32. Pagano D, Lewis ME, Townend JN, Davies P, Camici PG, Bonser RS. Coronary revascularization for postischemic heart failure: how myocardial viability affects survival. Heart. 1999;82:684-688.

33. Tamaki N, Kawamoto M, Takahashi N, et al. Prognostic value of an increase in fluorine-18 deoxyglucose uptake in patients with myocardial infarction: comparison with stress thallium imaging. J Am Coll Cardiol. 1993;22:1621-1627.

34. Kitsiou AN, Srinivasan G, Quyyumi AA, Summers RM, Bacharach SL, Dilsizian V. Stress-induced reversible and mild-to-moderate irreversible thallium defects: Are they equally accurate for predicting recovery of regional left ventricular function after revascularization? Circulation. 1998;98:501-508.

35. Gioia G, Powers J, Heo J, Iskandrian AS. Prognostic value of rest-redistribution tomographic thallium-201 imaging in ischemic cardiomyopathy. Am J Cardiol. 1995;75:759-762.

36. Pagley PR, Beller GA, Watson DD, Gimple LW, Ragosta M. Improved outcome after coronary bypass surgery in patients with ischemic cardiomyopathy and residual myocardial viability. Circulation. 1997;96:793-800.

37. Zafrir N, Leppo JA, Reinhardt CP, Dahlberg ST. Thallium reinjection versus standard stress/delay redistribution imaging for prediction of cardiac events. J Am Coll Cardiol. 1998;31:1280-1285.

38. Cuocolo A, Petretta M, Nicolai E, et al. Successful coronary revascularization improves prognosis in patients with previous myocardial infarction and evidence of viable myocardium at thallium-201 imaging. Eur J Nucl Med. 1998;25:60-68.

39. Schneider CA, Voth E, Gawlich S, et al. Significance of rest technetium- $99 \mathrm{~m}$ sestamibi imaging for the prediction of improvement of left ventricular dysfunction after Q wave myocardial infarction: importance of infarct location adjusted thresholds. J Am Coll Cardiol. 1998;32:648-654.

40. Sciagra R, Pellegri M, Pupi A, et al. Prognostic implications of Tc-99m sestamibi viability imaging and subsequent therapeutic strategy in patients with chronic coronary artery disease and left ventricular dysfunction. J Am Coll Cardiol. 2000;36:739-745.

41. Schinkel AFL, Bax JJ, Boersma E, et al. Assessment of residual myocardial viability in regions with chronic electrocardiographic Q-wave infarction. Am Heart J. 2002;144:865-869.

42. Bax JJ, Poldermans D, Elhendy A, et al. Improvement of left ventricular ejection fraction, heart failure symptoms and prognosis after revascularization in patients with chronic coronary artery disease and viable myocardium detected by dobutamine stress echocardiography. J Am Coll Cardiol. 1999;34:163-169.

43. Chaudhry FA, Tauke JT, Alessandrini RS, et al. Prognostic implications of myocardial contractile reserve in patients with coronary artery disease and left ventricular dysfunction. J Am Coll Cardiol. 1999;34:730-738. 
44. Senior R, Kaul S, Lahiri A. Myocardial viability on echocardiography predicts long-term survival after revascularization in patients with ischemic congestive heart failure. J Am Coll Cardiol. 1999;33:1848-1854.

45. Afridi I, Grayburn PA, Panza J, Oh JK, Zoghbi WA, Marwick TH. Myocardial viability during dobutamine echocardiography predicts survival in patients with coronary artery disease and severe left ventricular systolic dysfunction. J Am Coll Cardiol. 1998;32:921-926.

46. Meluzin J, Cerny J, Frelich M, et al. Prognostic value of the amount of dysfunctional but viable myocardium in revascularized patients with coronary artery disease and left ventricular dysfunction. J Am Coll Cardiol. 1998;32: 912-920.

47. Williams MJ, Odabashian J, Laurer MS, Thomas JD, Marwick TH. Prognostic value of dobutamine echocardiography in patients with left ventricular dysfunction. J Am Coll Cardiol. 1996;27:132-139.

48. Shimoni S, Frangogiannis NG, Aggeli CJ, et al. Microvascular structural correlates of myocardial contrast echocardiography in patients with coronary artery disease and left ventricular dysfunction: implications for the assessment of myocardial hibernation. Circulation. 2002;106:950-956.

49. Nagueh SF, Vaduganathan $\mathrm{P}$, Ali $\mathrm{N}$, et al. Identification of hibernating myocardium: comparative accuracy of myocardial contrast echocardiography, rest-redistribution thallium-201 tomography and dobutamine echocardiography. J Am Coll Cardiol. 1997;29:985-993.

50. DeFilippi CR, Willett DL, Irani WN, Eichhorn EJ, Velasco CE, Grayburn PA. Comparison of myocardial contrast echocardiography and low-dose dobutamine stress echocardiography in predicting recovery of left ventricular function after coronary revascularization in chronic ischemic heart disease. Circulation. 1995; 92:2863-2868.

51. Shimoni S, Frangogiannis NG, Aggeli CJ, et al. Identification of hibernating myocardium with quantitative intravenous myocardial contrast echocardiography: comparison with dobutamine echocardiography and thallium-201 scintigraphy. Circulation. 2003;107:538-544.

52. Gotte MJ, van Rossum AC, Twisk JWR, Kuijer JPA, Marcus JT, Visser CA. Quantification of regional contractile function after infarction: strain analysis superior to wall thickening analysis in discriminating infarct from remote myocardium. J Am Coll Cardiol. 2001;37:808-817.

53. Simonetti OP, Kim RJ, Fieno DS, et al. An improved MR imaging technique for the visualization of myocardial infarction. Radiology. 2001;218:215-223.

54. Baer FM, Voth E, Schneider CA, Theissen P, Schicha H, Sechtem U. Comparison of low-dose dobutamine-gradient-echo magnetic resonance imaging and positron emission tomography with $\left[{ }^{18} \mathrm{~F}\right]$ fluorodeoxyglucose in patients with chronic coronary artery disease: a functional and morphological approach to the detection of residual myocardial viability. Circulation. 1995;91:1006-1015.

55. Kim RJ, Fieno DS, Parrish TB, et al. Relationship of MRI delayed contrast enhancement to irreversible injury, infarct age, and contractile function. Circulation. 1999;100:1992-2002.

56. Kaandorp TA, Lamb HJ, van der Wall EE, de Roos A, Bax JJ. Cardiovascular MR to access myocardial viability in chronic ischaemic LV dysfunction. Heart. 2005;91:1359-1365.

57. Bove CM, DiMaria JM, Voros S, Conaway MR, Kramer CM. Dobutamine response and myocardial infarct transmurality: functional improvement after coronary artery bypass grafting-initial experience. Radiology. 2006;240:835841.

58. Kim RJ, Wu E, Rafael A, et al. The use of contrast-enhanced magnetic resonance imaging to identify reversible myocardial dysfunction. $N$ Engl J Med. 2000;343: 1445-1453.

59. Schvartzman PR, Srichai MB, Grimm RA, et al. Nonstress delayed-enhancement magnetic resonance imaging of the myocardium predicts improvement of function after revascularization for chronic ischemic heart disease with left ventricular dysfunction. Am Heart J. 2003;146:535-541.

60. Selvanayagam JB, Kardos A, Francis JM, et al. Value of delayed-enhancement cardiovascular magnetic resonance imaging in predicting myocardial viability after surgical revascularization. Circulation. 2004;110:1535-1541.

61. Selvanayagam JB, Jerosch-Herold M, Porto I, et al. Resting myocardial blood flow is impaired in hibernating myocardium: a magnetic resonance study of quantitative perfusion assessment. Circulation. 2005;112:3289-3296.

62. Carmichael BB, Setser RM, Stillman AE, et al. Effects of surgical ventricular restoration on left ventricular function: dynamic MR imaging. Radiology. 2006; 241:710-717.

63. Mahnken AH, Koos R, Katoh M, et al. Assessment of myocardial viability in reperfused acute myocardial infarction using 16-slice computed tomography in comparison to magnetic resonance imaging. J Am Coll Cardiol. 2005;45: 2042-2047.

64. Nikolaou K, Sanz J, Poon M, et al. Assessment of myocardial perfusion and viability from routine contrast-enhanced 16-detector-row computed tomography of the heart: preliminary results. Eur Radiol. 2005;15:864-871.

65. Panza JA, Dilsizian V, Laurienzo JM, Curiel RV, Katsiyiannis PT. Relation between thallium uptake and contractile response to dobutamine: implications regarding myocardial viability in patients with chronic coronary artery disease and left ventricular dysfunction. Circulation. 1995;91:990-998.

66. Bax JJ, Poldermans D, Schinkel AFL, et al. Perfusion and contractile reserve in chronic dysfunctional myocardium: relation to functional outcome after surgical revascularization. Circulation. 2002;106(suppl 1):I14-I18.

67. Perrone-Filardi P, Pace L, Prastaro M, et al. Assessment of myocardial viability in patients with chronic coronary artery disease: rest-4-hour-24-hour ${ }^{201} \mathrm{Tl}$ tomography versus dobutamine echocardiography. Circulation. 1996;94:2712-2719.

68. Beanlands RS, Hendry PJ, Masters RG, deKemp RA, Woodend K, Ruddy TD. Delay in revascularization is associated with increased mortality rate in patients with severe left ventricular dysfunction and viable myocardium on fluorine 18-fluorodeoxyglucose positron emission tomography imaging. Circulation. 1998;98(19 suppl):II51-II56.

69. Bax JJ, Schinkel AFL, Boersma E, et al. Early versus delayed revascularization in patients with ischemic cardiomyopathy and substantial viability: impact on outcome. Circulation. 2003;108(suppl 1):II39-II42.

70. Bax JJ, Schinkel AFL, Boersma E, et al. Extensive left ventricular remodeling does not allow viable myocardium to improve in left ventricular ejection fraction after revascularization and is associated with worse long-term prognosis. Circulation. 2004;110(suppl 1):II18-II22.

71. Beanlands RS, Ruddy TD, deKemp RA, et al. Positron emission tomography and recovery following revascularization (PARR-1): the importance of scar and the development of a prediction rule for the degree of recovery of left ventricular function. J Am Coll Cardiol. 2002;40:1735-1743. 